\title{
Association between Multimorbidity and Kidney Function among Patients with Non-Dialysis-Dependent CKD
}

\author{
Shigeru Tanaka ${ }^{1}$ Toshiaki Nakano ${ }^{1}$ Hiroto Hiyamuta ${ }^{1}$ Kazuhiko Tsuruya ${ }^{2}$ and Takanari Kitazono ${ }^{1}$ for the \\ FKR Study Collaboration Group
}

\begin{abstract}
${ }^{1}$ Department of Medicine and Clinical Science, Graduate School of Medical Sciences, Kyushu University, Fukuoka, Japan
${ }^{2}$ Department of Nephrology, Nara Medical University, Nara, Japan
\end{abstract}

Aim: Individuals with chronic kidney disease $(\mathrm{CKD})$ have a high prevalence of comorbidities, including cardiovascular disease (CVD) and its risk factors. However, epidemiological results to assess the association between multimorbidity and kidney function among the CKD population remains limited.

Methods: We performed a cross-sectional analysis of the association between 23 comorbid conditions and reduced kidney function in 4,476 patients with non-dialysis-dependent CKD enrolled in a multicenter cohort in Japan. Reduced kidney function was defined as an estimated glomerular filtration rate of $\leq 60 \mathrm{~mL} / \mathrm{min} / 1.73 \mathrm{~m}^{2}$.

Results: The mean age of patients was 67 years (male, 56.0\%). The prevalence of hypertension, diabetes mellitus, dyslipidemia, prior CVD, cancer, and bone fracture, which are the major comorbidities, was $83.3 \%$, $28.7 \%, 45.9 \%, 23.3 \%, 12.7 \%$, and $6.3 \%$, respectively. Multivariable-adjusted analyses revealed that age, male sex, hypertension, dyslipidemia, prior CVD, body mass index, urinary protein excretion, and underlying kidney disease were independent factors associated with reduced kidney function. Importantly, the odds ratios (ORs) for reduced kidney function increased linearly as the number of major comorbid conditions increased (OR for 1-2 conditions: $2.22,95 \%$ confidence interval $[\mathrm{CI}]: 1.65-2.97$; OR for 3-4 conditions: $3.04,95 \%$ CI: 2.12-4.37; OR for $\geq 5$ conditions: 4.37, 95\% CI: 1.75-10.9). The upward trend in OR was more pronounced with cardiovascular comorbidities but not significant with non-cardiovascular comorbidities.

Conclusions: In conclusion, we observed an independent association between cardiovascular comorbidity and its risk factors and reduced kidney function. The results of this study highlight the importance of managing multimorbidity among patients with CKD.

Key words: Aging, Comorbidities, Cardiovascular disease, Epidemiology

\section{Introduction}

The prevalence of chronic kidney disease (CKD) increases dramatically in the elderly, with $30 \%$ of males and $40 \%$ of females in the general Japanese population aged $65-69$ years having stage $3 \mathrm{CKD}^{1)}$. The number of patients with newly diagnosed endstage kidney disease (ESKD) will increase by approximately $8 \%$ annually, and the cumulative number of patients receiving renal therapy will double to 5.44 million by 2030 . This increase is expected to be particularly noticeable in Asian countries ${ }^{2,3)}$. Hence, CKD is increasingly recognized as a global health burden.

Epidemiological results suggest an increased risk of cardiovascular disease (CVD) morbidity and mortality in patients with $\mathrm{CKD}^{4-6)}$. Furthermore, a meta-analysis of clinical studies evaluated the significance of $\mathrm{CKD}$ and confirmed that a glomerular filtration rate (GFR) of $<60 \mathrm{~mL} / \mathrm{min} / 1.73 \mathrm{~m}^{2}$ and albuminuria are risk factors for all-cause mortality, cardiovascular death, and $\mathrm{ESKD}^{7,8)}$. Patients with

Address for correspondence: Kazuhiko Tsuruya, Department of Nephrology, Nara Medical University, 840 Shijo-cho, Kashihara, Nara 634-8521, Japan 
CKD have a high prevalence of comorbidities related to risk factors for CVD, and their management is more complicated. An association between the complexity of care and life expectancy for patients with reduced kidney function has been reported ${ }^{9)}$. Complexity in patients with CKD increases the number of medications for multiple comorbidities and may lead to a further decline in kidney function through adverse drug reactions and low adherence ${ }^{10-12)}$. Presently, it is less clear what types of comorbidities, including CVD, are closely associated with reduced kidney function in patients with non-dialysis-dependent CKD. Furthermore, the dose-response relationship between cumulative comorbidities or polypharmacy and reduced kidney function has not been fully confirmed. A better understanding of the comorbid burden of patients with $\mathrm{CKD}$ would be useful to define strategies to improve care for patients with CKD. However, epidemiological results to assess the association between multimorbidity and kidney function among the $\mathrm{CKD}$ population are still scarce; hence, the characteristics of comorbid burden in this population are unknown.

The Fukuoka Kidney disease Registry (FKR) Study is a prospective multicenter cohort study of approximately 4,500 Japanese patients with CKD under nephrologist care. In this study, we report the characteristics of enrolled patients and comorbid conditions at baseline. We aimed to examine the association between comorbidities, including cardiovascular risk profile, and reduced kidney function and to assess the impact of multimorbidity on kidney function in Japanese patients with CKD.

\section{Materials and Methods}

\section{Participant Selection and Recruitment}

The design of the FKR study has been described previously ${ }^{13)}$. A total of 4,476 patients with CKD aged $\geq 16$ years under nephrologist care at 12 sites were enrolled between January 2013 and March 2017. All patients who met the definition of CKD according to the Kidney Disease Improving Global Outcomes (KDIGO) clinical practice guidelines were included ${ }^{14)}$. We analyzed data from all enrolled patients as the final study population. The study protocol was approved by the Clinical Research Ethics Committee of the Institutional Review Board at Kyushu University (approval number 469-08) and the ethics committees at all participating institutions. Written informed consent was obtained from all patients at the start of the study. This study was registered in the University Hospital Medical Information Network clinical trial registry (UMIN000007988). This study was performed following the Declaration of Helsinki. Data analysis was conducted from December 2020 to February 2021.

\section{Clinical Parameters}

Demographic and clinical data were collected from all patients at the time of enrollment. Serum levels of cystatin $\mathrm{C}$, creatinine $(\mathrm{Cr})$, albumin, calcium, phosphorus, total cholesterol, high-density lipoprotein cholesterol (HDL-C), triglycerides (TG), iron, ferritin, and high-sensitivity $\mathrm{C}$-reactive protein (CRP); total iron-binding capacity; and plasma levels of intact parathyroid hormone (PTH), urine protein, and urine $\mathrm{Cr}$ were determined via central measurements using blood and urine samples collected at enrollment. Lowdensity lipoprotein cholesterol (LDL-C) was determined using the Friedewald formula for patients with a TG concentration of $<400 \mathrm{mg} / \mathrm{dL}$. For patients with a TG concentration of $\geq 400 \mathrm{mg} / \mathrm{dL}$, LDL-C values from the direct measurement method from medical records were adopted. The corrected serum calcium concentration calculation was based on the serum albumin concentration, which was in turn based on the Payne formula: corrected calcium concentration $(\mathrm{mg} / \mathrm{dL})=$ observed total calcium concentration $(\mathrm{mg} / \mathrm{dL})+(4.0-$ serum albumin concentration $[\mathrm{g} / \mathrm{dL}])$. CKD was defined and categorized as G1-G5 according to the status of estimated GFR (eGFR) using KDIGO clinical practice guidelines $\left.{ }^{15}, 16\right)$. eGFR was calculated using the Schwartz formula in patients aged $<18$ years and using the following formula in patients aged $>18$ years ${ }^{17-19)}$ : eGFR $\left(\mathrm{mL} / \mathrm{min} / 1.73 \mathrm{~m}^{2}\right)=194 \times \mathrm{Cr}^{-1.094} \times$ Age $^{-0.287}$ (if female, $\times 0.739$ ). Medications administered to the cohort participants included the following drugs; antihypertensive agents, lipid-lowering agents, uric acid-lowering agents, antiplatelet/anticoagulant agents, oral antidiabetic agents, insulin, erythropoietinstimulating agents, iron supplements, agents for bone mineral metabolism disorders, laxatives, sodium bicarbonate, uremic toxins and potassium adsorbents, thyroid medications, glucocorticoids, immunosuppressants, antidepressants, insomnia medications, non-steroidal anti-inflammatory drugs, proton pump inhibitors, and $\mathrm{H} 2$ blockers.

\section{Comorbidity Definition}

Hypertension was defined as a systolic blood pressure of $\geq 140 \mathrm{mmHg}$ or a diastolic blood pressure of $\geq 90 \mathrm{mmHg}$ and/or current use of antihypertensive agents. Diabetes mellitus was identified in subjects with a hemoglobin A1c value $\geq$ of $6.5 \%$ (National Glycohemoglobin Standardization Program) or diabetic nephropathy and/or current treatment with 
insulin or oral antihyperglycemic drugs. Dyslipidemia was defined as an LDL-C of $\geq 140 \mathrm{mg} / \mathrm{dL}$, an HDL-C of $<40 \mathrm{mg} / \mathrm{dL}$, a TG of $\geq 150 \mathrm{mg} / \mathrm{dL}$, or current lipid-lowering drug use. Prior CVD was defined as a composite history of ischemic heart disease, congestive heart failure, ischemic stroke, hemorrhagic stroke, peripheral artery disease (PAD), thoracic aortic aneurysm, and abdominal aortic aneurysm. Ischemic heart disease was defined as myocardial infarction, exertional angina pectoris, and vasospastic angina pectoris as diagnosed through coronary angiography and coronary heart disease with revascularization (coronary artery bypass graft surgery or percutaneous transluminal coronary angioplasty). A past history of congestive heart failure was defined by the attending physician, regardless of hospitalization. A history of stroke was defined as a permanent neurological deficit and imaging evidence of intracranial lesions using computed tomography and magnetic resonance imaging of the brain. PAD was defined as symptoms of Fontaine class II or higher (intermittent claudication and resting leg pain), an ankle-brachial index of $<0.9$, arterial stenosis via imaging diagnosis, or revascularization (surgery or percutaneous angioplasty of peripheral artery). The presence or absence of atrial fibrillation was determined on the basis of medical records without distinguishing the type of atrial fibrillation (persistent or paroxysmal). Sleep apnea was diagnosed by simple/all-night polysomnography or treatment. The exclusion criteria included active malignancies; thus, a history of malignancy at baseline was defined as diagnosis and treatment on the basis of clinical symptoms, imaging, and histological evidence, and $>2$ years after the completion of treatment. Dementia and depression were diagnosed by the attending physician and in patients taking antidementia or antidepressant medications, respectively. Liver disease was defined as mild or severe liver dysfunction in hepatitis virus carriers, patients with chronic hepatitis or cirrhosis, and patients receiving antiviral treatment. A history of thyroid disease was defined as a diagnosis of Hashimoto's or Graves' disease or receipt of antithyroid medication or thyroid hormone replacement. A history of other comorbidities, including bone fracture, chronic obstructive pulmonary disease, and collagen disease, was identified on the basis of all available clinical data collected by clinical research coordinators (CRCs). Clinical information on each patient's health status and medication was collected from medical records using a structured data format by the CRCs in our research network. Comorbidity was evaluated using the Charlson Comorbidity Index ${ }^{20}$. We determined the 23 selected comorbidities for analysis on the basis of the components of the Charlson Comorbidity
Index and disease information available in this cohort.

\section{Statistical Analysis}

Continuous variables are presented as median and interquartile range, whereas categorical data are presented as number and percentage. The clinical backgrounds categorized as G1-G5 according to KDIGO clinical practice guidelines were compared using trend analyses; a linear regression model was used for categorical variables and a logistic regression model for continuous variables. To determine independent associated factors of a reduced GFR, we selected covariates using a logistic regression model and a stepwise backward elimination with a $P$ value of $<0.05$ for the remaining variables. In this selection, the clinically or biologically plausible risk factors for reduced GFR were included as initial candidate variables, including age, sex, smoking habit, alcohol intake, underlying kidney disease (hypertensive nephrosclerosis, diabetic nephropathy, and others), hypertension, diabetes mellitus, dyslipidemia, prior history of CVD, cancer, bone fracture, body mass index (BMI), and urinary protein-to-Cr ratio (UPCR). The final model consisted of age, sex, underlying kidney disease, hypertension, diabetes mellitus, dyslipidemia, history of CVD, BMI, and UPCR. Multivariable-adjusted odds ratios (ORs) and 95\% confidence intervals (CIs) for the prevalence of reduced kidney function $\left(<60 \mathrm{~mL} / \mathrm{min} / 1.73 \mathrm{~m}^{2}\right)$ were estimated using this final model. Variables relevant to the categories were excluded from each model. We also plotted a box-and-whisker diagram showing the association between the number of comorbidities and eGFR and tested this association in a multivariable-adjusted linear regression model with eGFR as the dependent variable. To assess the effect of the number of medications on reduced kidney function, we evaluated the multivariate ORs for reduced eGFR in each of the categorical groups classified according to the number of medications ( 0 , 1-2, 3-4, 5-6, and $\geq 7$ ). Statistical analyses were undertaken using SAS v9.4 (SAS Institute, Cary, NC, USA) and STATA v16 (Stata, College Station, TX, USA). A $P$ value of $<0.05$ (two-tailed) was considered statistically significant.

\section{Results}

\section{Demographics and Baseline Characteristics}

Table 1 presents the patients' baseline characteristics according to CKD stages. The mean age of patients was 67 (15.9) years (female, 44.0\%). The distribution of CKD stages was 259 (5.8\%), 825 (18.4\%), 834 (18.6\%), 934 (20.9\%), 1,093 (24.4\%), 
Table 1. General characteristics of patients according to CKD stages

\begin{tabular}{|c|c|c|c|c|c|c|c|}
\hline & All patients & Stage G1-2 & Stage G3a & Stage G3b & Stage G4 & Stage G5 & $P$ value \\
\hline No. of patients & 4,476 & 1,084 & 834 & 934 & 1,093 & 531 & \\
\hline Age, years & $67(55-76)$ & $50(38-65)$ & $65(56-73)$ & $70(62-78)$ & $73(64-80)$ & $72(64-79)$ & $<0.001$ \\
\hline Sex (female), \% & 44.0 & 55.5 & 45.9 & 36.8 & 38.8 & 40.7 & $<0.001$ \\
\hline \multicolumn{8}{|l|}{ Smoking history } \\
\hline Never smokers, $\%$ & 47.6 & 12.9 & 9.3 & 9.5 & 11.4 & 12.1 & $<0.001$ \\
\hline Current smokers, $\%$ & 11.2 & 57.9 & 52.1 & 45.9 & 41.5 & 36.6 & 0.57 \\
\hline Former smokers, $\%$ & 41.2 & 29.1 & 38.6 & 44.5 & 47.1 & 51.3 & $<0.001$ \\
\hline \multicolumn{8}{|l|}{ Drinking history } \\
\hline Never drinkers, \% & 38.8 & 37.2 & 36.8 & 38.1 & 40.8 & 29.9 & 0.02 \\
\hline Current drinkers, $\%$ & 48.6 & 55.3 & 55.6 & 47.8 & 43.0 & 56.0 & $<0.001$ \\
\hline Former drinkers, $\%$ & 12.6 & 7.5 & 7.6 & 14.2 & 16.3 & 14.2 & $<0.001$ \\
\hline \multicolumn{8}{|l|}{ Underlying kidney disease } \\
\hline Biopsy proven primary CGN, $\%$ & 34.9 & 11.5 & 13.7 & 16.9 & 16.0 & 19.0 & $<0.001$ \\
\hline CGN without biopsy, $\%$ & 14.4 & 70.4 & 44.2 & 32.8 & 13.0 & 7.3 & $<0.001$ \\
\hline Diabetic nephropathy, $\%$ & 11.2 & 1.4 & 5.6 & 10.6 & 17.9 & 30.9 & $<0.001$ \\
\hline Hypertensive nephrosclerosis, $\%$ & 21.6 & 4.0 & 16.7 & 36.8 & 33.9 & 26.2 & $<0.001$ \\
\hline Other, $\%$ & 17.9 & 12.7 & 19.8 & 2.8 & 19.2 & 16.6 & 0.80 \\
\hline \multicolumn{8}{|l|}{ Anthropometric data } \\
\hline Height, $\mathrm{cm}$ & $160(154-167)$ & $161(155-168)$ & $161(154-168)$ & $161(154-167)$ & $160(153-166)$ & $160(153-166)$ & $<0.001$ \\
\hline Body weight, kg & $59(51.3-67.6)$ & $58.5(51.6-68.5)$ & $60.3(52-68.6)$ & $60.5(52-68)$ & $58(50-67)$ & $58(50-65)$ & $<0.001$ \\
\hline $\mathrm{BMI}, \mathrm{kg} / \mathrm{m}^{2}$ & $22.9(20.6-25.6)$ & $22.6(20.3-25.9)$ & $23.2(20.9-26.0)$ & $23.2(21.0-25.7)$ & $22.8(20.5-25.4)$ & $22.6(20.4-24.9)$ & 0.05 \\
\hline \multicolumn{8}{|l|}{ Blood pressure } \\
\hline Systolic, mmHg & $130(119-142)$ & $124(113-135)$ & $130(120-140)$ & $130(120-142)$ & $132(121-144)$ & $135(126-147)$ & $<0.001$ \\
\hline Diastolic, $\mathrm{mmHg}$ & $74(67.3-82)$ & $74(66-82)$ & $76(70-84)$ & $74(68-82)$ & $73(65-81)$ & $72(65-80)$ & $<0.001$ \\
\hline Blood pressure $<130 / 80 \mathrm{mmHg}, \%$ & 41.7 & 54.6 & 41.0 & 39.8 & 37.1 & 29.4 & $<0.001$ \\
\hline Blood pressure $<140 / 90 \mathrm{mmHg}, \%$ & 68.5 & 79.2 & 69.5 & 67.9 & 64.2 & 54.8 & $<0.001$ \\
\hline \multicolumn{8}{|l|}{ Kidney parameters } \\
\hline Serum Cr, mg/dL & $1.29(0.88-2.09)$ & $0.70(0.61-0.83)$ & $1.02(0.87-1.15)$ & $1.36(1.22-1.54)$ & $2.13(1.83-2.50)$ & $4.09(3.53-4.98)$ & $<0.001$ \\
\hline Serum cystatin $C, m g / L$ & $1.43(0.96-2.26)$ & $0.79(0.69-0.92)$ & $1.08(0.97-1.23)$ & $1.49(1.30-1.72)$ & $2.30(1.97-2.62)$ & $3.53(3.10-4.00)$ & $<0.001$ \\
\hline $\mathrm{eGFR}, \mathrm{mL} / \mathrm{min} / 1.73 \mathrm{~m}^{2}$ & $40.1(23.4-59.2)$ & $76.9(67.8-89.2)$ & $51.5(48.0-55.3)$ & $37.3(33.9-41.5)$ & $22.8(19.1-26.4)$ & $11.3(9.2-13.2)$ & $<0.001$ \\
\hline UPCR, $g / g C r$ & $0.40(0.11-1.30)$ & $0.16(0.07-0.53)$ & $0.17(0.07-0.64)$ & $0.31(0.10-1.03)$ & $0.69(0.27-1.93)$ & $1.83(0.93-3.54)$ & $<0.001$ \\
\hline UACR, $\mathrm{mg} / \mathrm{gCr}$ & $206.5(35.7-794.5)$ & $76.6(15.2-348.3)$ & $84.5(16.7-396.6)$ & $158.2(30.6-645.9)$ & $381(108.8-1119.1)$ & $942.3(430.6-2070.1)$ & $<0.001$ \\
\hline \multicolumn{8}{|l|}{$\begin{array}{l}\text { Lipids, uric acid, hemoglobin Alc, CRP, } \\
\text { and albumin }\end{array}$} \\
\hline Total cholesterol, mg/dL & $191(167-217)$ & $199(178-223)$ & $198(175-221)$ & $163(169-217)$ & $183(158-212)$ & $172(149-199)$ & $<0.001$ \\
\hline $\mathrm{LDL}-\mathrm{C}, \mathrm{mg} / \mathrm{dL}$ & $104.4(84.7-125.8)$ & $109(91.8-129.8)$ & $108.6(89.8-129.6)$ & $105.4(86.4-125 / 0)$ & $100.2(80.0-122.0)$ & $91.7(71.4-115.2)$ & $<0.001$ \\
\hline HDL-C, $\mathrm{mg} / \mathrm{dL}$ & $56(45-70)$ & $63(52-77)$ & $58(47-72)$ & $56(12-69)$ & $52(10-66)$ & $49(39-60)$ & 0.01 \\
\hline Triglycerides, $\mathrm{mg} / \mathrm{dL}$ & $120(87-171)$ & $107(77-156)$ & $122(87-174)$ & $125(91-170)$ & $125(91-180)$ & $126(92-168)$ & $<0.001$ \\
\hline Uric acid, $\mathrm{mg} / \mathrm{dL}$ & $6.1(5.2-7.1)$ & $5.4(4.5-6.4)$ & $6.1(5.3-6.9)$ & $6.4(5.6-7.3)$ & $6.4(5.6-7.5)$ & $6.6(5.6-7.7)$ & $<0.001$ \\
\hline Hemoglobin Alc, \% & $6.0(5.6-6.4)$ & $5.8(5.5-6.2)$ & $6.0(5.6-6.4)$ & $6.0(5.7-6.5)$ & $6.1(5.7-6.6)$ & $5.9(5.2-6.5)$ & $<0.001$ \\
\hline $\mathrm{CRP}, \mathrm{mg} / \mathrm{dL}$ & $0.05(0.02-0.12)$ & $0.04(0.02-0.09)$ & $0.05(0.02-0.11)$ & $0.06(0.03-0.13)$ & $0.07(0.03-0.15)$ & $0.06(0.03-0.16)$ & 0.01 \\
\hline Serum albumin, $g / d L$ & $4.1(3.8-4.3)$ & $4.2(4.0-4.5)$ & $4.2(3.9-4.4)$ & $4.1(3.8-4.3)$ & $3.9(3.7-4.2)$ & $3.8(3.5-4.1)$ & $<0.001$ \\
\hline \multicolumn{8}{|l|}{ Anemia parameters and iron status } \\
\hline Hemoglobin, $g / d L$ & $12.7(11.3-14.1)$ & $13.9(13.0-15.0)$ & $13.7(12.6-14.8)$ & $12.8(11.6-14.1)$ & $11.6(10.7-12.7)$ & $10.8(10.1-11.7)$ & $<0.001$ \\
\hline Iron, $\mu \mathrm{g} / \mathrm{mL}$ & $84(65-106)$ & $90(68-115)$ & $93(71-113)$ & $84(66-106)$ & $78(61-95)$ & $77(59-94)$ & $<0.001$ \\
\hline Transferrin saturation, $\%$ & $28.6(22.0-36.1)$ & $28.4(21.6-36.5)$ & $30.3(22.7-37.1)$ & $28.3(21.9-36.0)$ & $27.9(21.6-35.0)$ & $29.8(22.9-36.8)$ & 0.87 \\
\hline Ferritin, ng/mL & $100(46.1-186)$ & $77.4(29.3-157)$ & $94.7(48.2-171)$ & $99(47.3-181)$ & $109(51.6-198)$ & $137(75.9-248)$ & $<0.001$ \\
\hline \multicolumn{8}{|l|}{ MBD parameters and electrolytes } \\
\hline Corrected calcium, mg/dL & $9.3(9.0-9.5)$ & $9.2(9.0-9.5)$ & $9.3(9.1-9.5)$ & $9.4(9.1-9.6)$ & $9.3(9.0-9.6)$ & $9.0(8.6-9.4)$ & $<0.001$ \\
\hline Phosphorus, $\mathrm{mg} / \mathrm{dL}$ & $3.4(3.0-3.9)$ & $3.3(2.9-3.7)$ & $3.3(2.9-3.7)$ & $3.3(2.9-3.7)$ & $3.5(3.1-3.9)$ & $4.2(3.3-4.8)$ & $<0.001$ \\
\hline Intact PTH, $\mathrm{pg} / \mathrm{mL}$ & $67(46-110)$ & $48(37-62)$ & $55(42-71)$ & $65(48-88)$ & $104(71.5-152)$ & $221(141-334)$ & $<0.001$ \\
\hline Serum sodium, $\mathrm{mEq} / \mathrm{L}$ & $141(139-142)$ & $141(140-142)$ & $141(140-142)$ & $141(139-142)$ & $141(139-142)$ & $140(138-142)$ & $<0.001$ \\
\hline Serum potassium, $\mathrm{mEq} / \mathrm{L}$ & $4.4(4.1-4.7)$ & $4.1(3.9-4.4)$ & $4.3(4.0-4.6)$ & $4.4(4.1-4.7)$ & $4.6(4.3-4.9)$ & $4.7(4.3-5.0)$ & $<0.001$ \\
\hline
\end{tabular}

Abbreviations: CKD, chronic kidney disease; CGN, chronic glomerulonephritis; BMI, body mass index; Cr, creatinine; eGFR, estimated glomerular filtration rate; UPCR, urinary protein-to-creatinine ratio; UACR, urinary albumin-to-creatinine ratio; LDL-C, low-density lipoprotein cholesterol; HDL-C, high-density lipoprotein cholesterol; CRP, C-reactive protein; MBD, mineral bone disorders; PTH, parathyroid hormone. Values are presented as median (interquartile range) or percentage. 


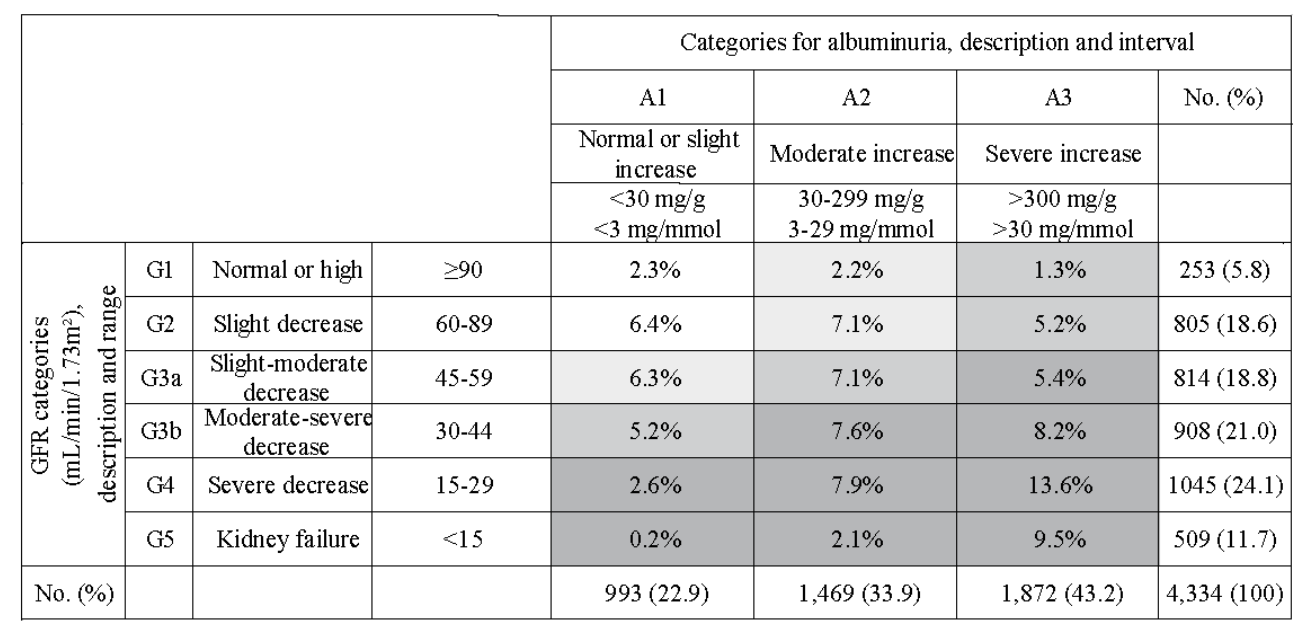

\begin{tabular}{|l|l|}
\hline & Low risk (if no other markers of CKD) \\
& Moderate risk \\
\cline { 1 - 2 } High risk \\
\cline { 1 - 2 } Very high risk
\end{tabular}

Fig. 1. Classification of chronic kidney disease using GFR and ACR categories (KDIGO 2012)

Abbreviations: GFR, glomerular filtration rate; ACR, albumin-to-creatinine rate.

and $531(11.9 \%)$ for G1, G2, G3a, G3b, G4, and G5, respectively. The percentage of former smokers increased with CKD progression, whereas the percentage of never smokers decreased. The proportion of drinkers increased with CKD progression in both former and current smokers. Of the 4,476 patients, 2,204 patients (49.3\%) had chronic glomerulonephritis (CGN), and 1,561 patients (34.9\%) had biopsyproven CGN. Other causes of kidney disease included diabetic nephropathy in 502 patients $(11.2 \%)$ and hypertensive nephrosclerosis in 969 patients $(21.6 \%)$, whereas the remaining 801 patients $(17.9 \%)$ had other causes. In the earlier CKD stages (G1 and G2), $81.9 \%$ of patients had CGN as the cause of kidney disease. By contrast, the proportion of patients with diabetic nephropathy and hypertensive nephrosclerosis as the cause of kidney disease increased to $30.9 \%$ and $26.2 \%$ in stage G5, respectively. The mean BMI was $22.9 \mathrm{~kg} / \mathrm{m}^{2}$. The mean systolic and diastolic blood pressures of patients were 130 and $74 \mathrm{mmHg}$, respectively.

The median eGFR was $40.1 \mathrm{~mL} / \mathrm{min} / 1.73 \mathrm{~m}^{2}$. The median UPCR and median urinary albuminto-Cr ratio were $0.40 \mathrm{~g} / \mathrm{gCr}$ and $206.5 \mathrm{mg} / \mathrm{gCr}$, respectively. The group with a lower eGFR had lower total cholesterol, LDL-C, and HDL-C levels but higher TG levels. There was a trend toward higher uric acid and CRP levels and lower albumin levels in the low eGFR group compared with the high eGFR group. The low eGFR group had lower levels of hemoglobin, serum iron, and calcium but higher levels of ferritin, phosphorus, intact PTH, and potassium. Use of antihypertensives, lipid-lowering drugs, insulin, urate-lowering drugs, antiplatelet/ anticoagulants, erythropoiesis-stimulating agents, mineral bone disorders-related drugs, uremic toxins, and potassium adsorbents was higher in the low eGFR group compared with the high eGFR group (Supplementary Table 1). Fig. 1 shows the distribution of enrolled patients stratified by eGFR and albuminuria ${ }^{10)}$. Risk rankings for adverse outcomes based on eGFR and albuminuria levels stratified 8.6\%, $15.6 \%, 18.7 \%$, and $57.0 \%$ of the total cohort as mild, moderate, severe, and very severe, respectively. As Fig. 2 shows, the number of medications increased linearly with the progression of the CKD stage, and a significant dose-response relationship between polypharmacy and reduced eGFR was observed in the multivariable-adjusted model (Table 6).

Independent Associated Factors of Reduced Kidney Function

Table 2 lists the multivariable independent associated factors of an eGFR $<60 \mathrm{~mL} / \mathrm{min} / 1.73 \mathrm{~m}^{2}$. Logistic regression revealed that older age (OR for 10 year increment, $1.78 ; 95 \% \mathrm{CI}, 1.68-1.90)$, male sex (OR, 1.25; 95\% CI, 1.05-1.50), underlying kidney disease (OR for others, 3.46; 95\% CI: 2.74-4.38; OR for hypertensive nephrosclerosis, 5.94; 95\% CI, 4.228.37; and OR for diabetic nephropathy, 9.59; $95 \%$ CI, 5.39-17.1), history of CVD (OR, 1.68; 95\% CI, 1.26-2.40), hypertension (OR, 2.68; 95\% CI, 2.15- 


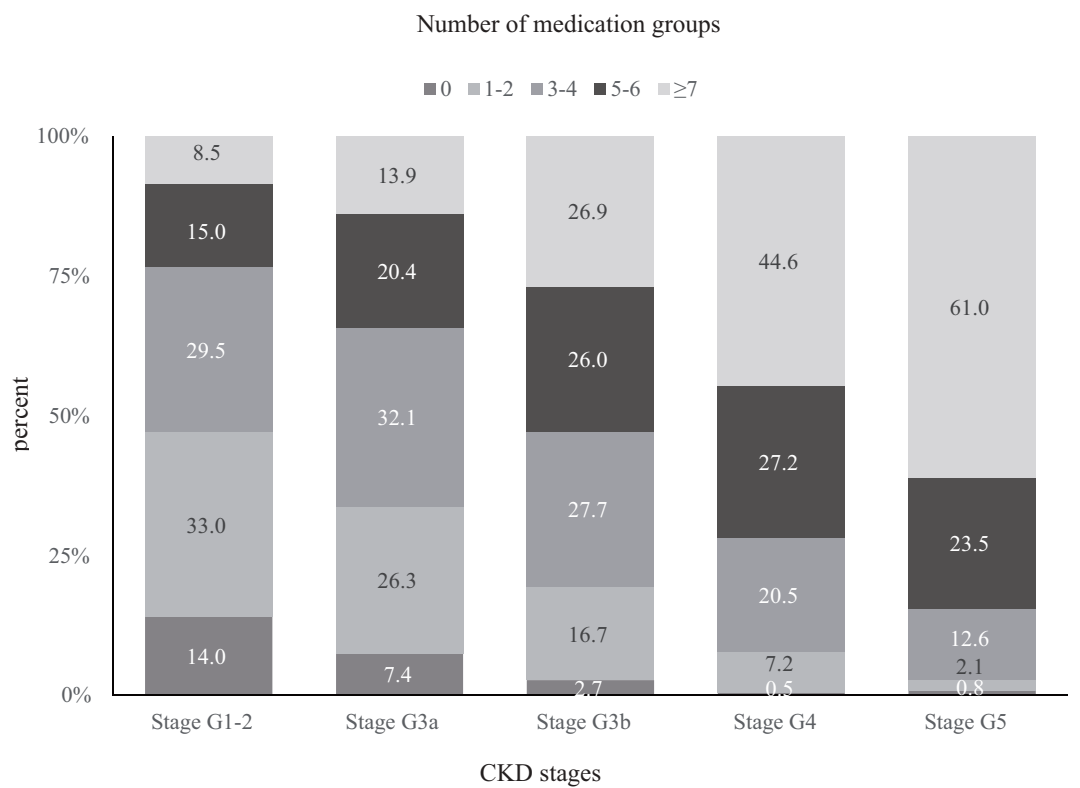

Fig. 2. Distribution of the number of CKD-related medications by CKD stages Abbreviation: CKD, chronic kidney disease.

Table 2. Multivariable independent associated factors for estimated glomerular filtration rate less than $60 \mathrm{~mL} / \mathrm{min}$ per $1.73 \mathrm{~m}^{2}$

\begin{tabular}{lcr}
\hline Variable & Adjusted odds ratio (95\% CI) & $P$ value \\
\hline Age (per 10-year increment) & $1.78(1.68-1.90)$ & $<0.001$ \\
Men (vs. women) & $1.25(1.05-1.50)$ & 0.012 \\
Underlying kidney disease & & $<0.001$ \\
$\quad$ Others (vs. CGN) & $3.46(2.74-4.38)$ & $<0.001$ \\
$\quad$ Hypertensive nephrosclerosis (vs. CGN) & $5.94(4.22-8.37)$ & $<0.001$ \\
$\quad$ Diabetic nephropathy (vs. CGN) & $9.59(5.39-17.1)$ & $<0.001$ \\
Cardiovascular disease (vs. without) & $1.68(1.26-2.40)$ & $<0.001$ \\
Hypertension (vs. without) & $2.68(2.15-3.34)$ & 0.005 \\
Diabetes mellitus (vs. without) & $0.71(0.56-0.90)$ & 0.008 \\
Dyslipidemia (vs. without) & $1.28(1.07-1.54)$ & 0.001 \\
Body mass index (per 5-kg/m ${ }^{2}$ decrement) & $1.20(1.07-1.34)$ & $<0.001$ \\
Urinary protein excretion (per 1-g/gCr increment) & $1.47(1.34-1.62)$ & \\
\hline
\end{tabular}

Abbreviations: CGN, chronic glomerulonephritis; CI, confidence interval.

Hypertension was defined as a systolic blood pressure $\geq 140 \mathrm{mmHg}$ or a diastolic blood pressure $\geq 90 \mathrm{mmHg}$ and/or current use of antihypertensive agents.

Diabetes mellitus was identified in patients with a hemoglobin A1c value $\geq 6.5 \%$ (National Glycohemoglobin Standardization Program), whose underlying kidney disease was diabetic nephropathy, and/or who were receiving treatment with insulin or oral antihyperglycemic drugs.

Dyslipidemia was defined as a triglyceride concentration $\geq 150 \mathrm{mg} / \mathrm{dL}$, a low-density lipoprotein cholesterol concentration $\geq$ $140 \mathrm{mg} / \mathrm{dL}$, a high-density lipoprotein cholesterol concentration $<40 \mathrm{mg} / \mathrm{dL}$, or use of lipid-lowering medication.

Cardiovascular disease was defined as a composite of ischemic heart disease, congestive heart failure, ischemic stroke, hemorrhagic stroke, peripheral artery disease, thoracic aortic aneurysm, and abdominal aortic aneurysm.

${ }^{a}$ Variables were selected by a logistic regression model using a stepwise backward method with $P<0.05$ for remaining variables to determine the independent associated factors for estimated glomerular filtration rate less than $60 \mathrm{~mL} / \mathrm{min}$ per $1.73 \mathrm{~m}^{2}$.

3.34), diabetes mellitus (OR, 0.71; 95\% CI, 0.56$0.90)$, dyslipidemia (OR, 1.28; 95\% CI, 1.07-1.54), lower BMI (OR for $5 \mathrm{~kg} / \mathrm{m}^{2}$ decrement, $1.20 ; 95 \%$
CI, 1.07-1.34), and higher UPCR (OR for $1 \mathrm{~g} / \mathrm{gCr}$ increment, $1.47 ; 95 \%$ CI, 1.34-1.62) were independently associated with a reduced eGFR. 
Table 3. Prevalence of major comorbid conditions of patients according to CKD stages

\begin{tabular}{|c|c|c|c|c|c|c|c|}
\hline & All patients & Stage G1-2 & Stage G3a & Stage G3b & Stage G4 & Stage G5 & $P$ value \\
\hline Hypertension, \% & 83.3 & 63.0 & 81.8 & 90.8 & 95.3 & 96.4 & $<0.001$ \\
\hline Diabetes mellitus, \% & 28.7 & 15.5 & 20.4 & 29.2 & 38.4 & 47.7 & $<0.001$ \\
\hline Dyslipidemia, \% & 45.9 & 39.9 & 46.9 & 47.1 & 49.4 & 50.1 & $<0.001$ \\
\hline Cardiovascular disease, $\%$ & 23.3 & 6.7 & 14.7 & 25.5 & 37.9 & 36.6 & $<0.001$ \\
\hline Ischemic heart disease, $\%$ & 10.8 & 2.7 & 7.3 & 12.6 & 16.4 & 17.9 & $<0.001$ \\
\hline Congestive heart failure, $\%$ & 3.0 & 0.3 & 0.4 & 3.5 & 5.7 & 5.9 & $<0.001$ \\
\hline Ischemic stroke, $\%$ & 9.1 & 2.9 & 5.9 & 8.7 & 15.7 & 14.2 & $<0.001$ \\
\hline Hemorrhagic stroke, \% & 2.0 & 0.7 & 1.1 & 2.4 & 3.5 & 2.6 & $<0.001$ \\
\hline Peripheral artery disease, $\%$ & 3.2 & 0.9 & 1.1 & 3.6 & 5.1 & 6.6 & $<0.001$ \\
\hline Thoracic aortic aneurysm, \% & 0.9 & 0.0 & 0.7 & 1.0 & 1.6 & 1.3 & $<0.001$ \\
\hline Abdominal aortic aneurysm, $\%$ & 2.1 & 0.4 & 0.6 & 2.5 & 3.5 & 4.5 & $<0.001$ \\
\hline Cancer, $\%$ & 12.7 & 5.3 & 11.3 & 17.5 & 15.8 & 15.1 & $<0.001$ \\
\hline Bone fracture, $\%$ & 6.3 & 4.1 & 6.2 & 6.3 & 6.9 & 9.8 & $<0.001$ \\
\hline \multicolumn{8}{|l|}{ Frequency (no. of conditions) } \\
\hline 0 & 7.4 & 21.7 & 8.0 & 3.2 & 1.9 & 0.8 & $<0.001$ \\
\hline $1-2$ & 59.7 & 66.2 & 68.6 & 60.3 & 50.7 & 46.1 & $<0.001$ \\
\hline $3-4$ & 28.9 & 11.5 & 22.6 & 32.0 & 40.3 & 44.6 & $<0.001$ \\
\hline$\geq 5$ & 4.0 & 0.6 & 0.8 & 4.5 & 7.1 & 8.5 & $<0.001$ \\
\hline Charlson Comorbidity Index & $1.16(1.45)$ & $0.51(0.98)$ & $0.84(1.21)$ & $1.27(1.43)$ & $1.65(1.60)$ & $1.79(1.61)$ & $<0.001$ \\
\hline
\end{tabular}

Abbreviations: CKD, chronic kidney disease. Values are presented as mean (standard deviation) or percentage.

Hypertension was defined as a systolic blood pressure $\geq 140 \mathrm{mmHg}$ or a diastolic blood pressure $\geq 90 \mathrm{mmHg}$ and/or current use of antihypertensive agents. Diabetes mellitus was identified in patients with a hemoglobin A1c value $\geq 6.5 \%$ (National Glycohemoglobin Standardization Program), whose underlying kidney disease was diabetic nephropathy, and/or who were receiving treatment with insulin or oral antihyperglycemic drugs.

Dyslipidemia was defined as a triglyceride concentration $\geq 150 \mathrm{mg} / \mathrm{dL}$, a low-density lipoprotein cholesterol concentration $\geq 140 \mathrm{mg} / \mathrm{dL}$, a high-density lipoprotein cholesterol concentration $<40 \mathrm{mg} / \mathrm{dL}$, or use of lipid-lowering medication. Cardiovascular disease was defined as a composite of ischemic heart disease, congestive heart failure, ischemic stroke, hemorrhagic stroke, peripheral artery disease, thoracic aortic aneurysm, and abdominal aortic aneurysm.

\section{Comorbidities Associated with Reduced Kidney Function}

As Table 3 shows, the prevalence of major comorbid conditions, including hypertension, diabetes mellitus, dyslipidemia, prior CVD, cancer, and bone fracture was $3,727(83.3 \%), 1,285(28.7 \%), 2,055$ (45.9\%), $1,041(23.3 \%), 567(12.7 \%)$, and 282 (6.3\%), respectively. The proportion of patients with these comorbidities was significantly increased in the group with advanced CKD (Supplementary Table 2). Importantly, the cumulative number of comorbidities increased, and the Charlson Comorbidity Index increased in the population with a reduced eGFR ( $P$ for trend, $<0.001)$. Table 4 shows the prevalence and association of comorbidities with an eGFR of $<60$ $\mathrm{mL} / \mathrm{min} / 1.73 \mathrm{~m}^{2}$. Of all cardiovascular comorbidities, hypertension, diabetes mellitus, dyslipidemia, ischemic heart disease, and congestive heart failure were associated with elevated ORs with an eGFR of $<60$ $\mathrm{mL} / \mathrm{min} / 1.73 \mathrm{~m}^{2}$. For non-cardiovascular comorbidities, cancer was a significant risk factor for decreased kidney function, whereas collagen disease and liver disease were associated with lowered ORs with an eGFR of $<60 \mathrm{~mL} / \mathrm{min} / 1.73 \mathrm{~m}^{2}$. Sensitivity analysis revealed a clearer dose-response relationship between reduced GFR and comorbidity in the model using an eGFR of $<45 \mathrm{~mL} / \mathrm{min} / 1.73 \mathrm{~m}^{2}$ as the dependent variable (Supplementary Table 3). Supplementary Fig. 1 shows the dose-response association between eGFR and the number of comorbidities. Furthermore, a significant association between eGFR and comorbidity categories was identified in the multivariable-adjusted linear regression model with eGFR as the dependent variable (Supplementary Table 4).

\section{Multimorbidity and Reduced Kidney Function}

Table 5 demonstrates the association between multimorbidity and reduced eGFR. Compared with the reference group without any comorbidities, adjusted ORs increased linearly as the cumulative number of conditions increased (all 23 registered comorbidities: OR for $1-2$ conditions, $2.11 ; 95 \% \mathrm{CI}$, 1.54-3.41; OR for 3-4 conditions, 2.38; 95\% CI, 1.66-3.41; and OR for $\geq 5$ conditions, 2.94 ; $95 \%$ CI: 1.74-4.97; and major comorbidities: OR for 1-2 conditions, 2.22; 95\% CI, 1.65-2.97; OR for 3-4 conditions, 3.04; 95\% CI, 2.12-4.37; and OR for $\geq 5$ 
Table 4. Comorbidities associated with an eGFR $<60 \mathrm{~mL} / \mathrm{min} / 1.73 \mathrm{~m}^{2}$

\begin{tabular}{|c|c|c|c|c|}
\hline Comorbidities & $\begin{array}{c}\text { No. (\%) of included } \\
\text { subjects }\end{array}$ & $\begin{array}{c}\text { Prevalence of subjects } \\
\text { with an eGFR }<60 \\
\mathrm{~mL} / \mathrm{min} / 1.73 \mathrm{~m}^{2}(\%)\end{array}$ & $\begin{array}{l}\text { Adjusted odds ratio } \\
\qquad(95 \% \mathrm{CI})\end{array}$ & $P$ value \\
\hline \multicolumn{5}{|l|}{ Cardiovascular risk factors } \\
\hline Hypertension (vs. without) & $3,727(83.3)$ & 81.9 & $2.68(2.15-3.34)$ & $<0.001$ \\
\hline Diabetes mellitus (vs. without) & $1,285(28.7)$ & 86.9 & $0.71(0.56-0.90)$ & 0.005 \\
\hline Dyslipidemia (vs. without) & $2,055(45.9)$ & 79.1 & $1.28(1.07-1.54)$ & 0.01 \\
\hline Sleep apnea syndrome (vs. without) & $61(1.4)$ & 83.6 & $1.04(0.46-2.33)$ & 0.93 \\
\hline Atrial fibrillation (vs. without) & $282(5.5)$ & 92.2 & $1.08(0.63-1.85)$ & 0.79 \\
\hline Cardiovascular disease (vs. without) & $1,041(23.3)$ & 93.0 & $1.68(1.26-2.40)$ & $<0.001$ \\
\hline Ischemic heart disease (vs. without) & $481(10.8)$ & 94.0 & $1.63(1.05-2.51)$ & 0.03 \\
\hline Congestive heart failure (vs. without) & $132(3.0)$ & 97.7 & $5.04(1.52-16.7)$ & 0.01 \\
\hline Ischemic stroke (vs. without) & $408(9.1)$ & 92.4 & $1.40(0.92-2.14)$ & 0.12 \\
\hline Hemorrhagic stroke (vs. without) & $90(2.0)$ & 92.2 & $1.62(0.70-3.75)$ & 0.26 \\
\hline Peripheral artery disease (vs. without) & $144(3.2)$ & 93.1 & $1.18(0.53-2.63)$ & 0.68 \\
\hline Thoracic aortic aneurysm (vs. without) & $39(0.9)$ & $92.3^{\mathrm{a}}$ & $1.85(0.53-6.47)^{\mathrm{a}}$ & $0.33^{\mathrm{a}}$ \\
\hline Abdominal aortic aneurysm (vs. without) & $94(2.1)$ & 95.7 & $1.63(0.56-4.61)$ & 0.38 \\
\hline Non-cardiovascular disease (vs. without) & $3,392(75.8)$ & 83.3 & $1.01(0.83-1.23)$ & 0.93 \\
\hline Cancer (vs. without) & $567(12.7)$ & 90.0 & $1.41(1.02-1.94)$ & 0.04 \\
\hline Bone fracture (vs. without) & $282(6.3)$ & 84.4 & $0.93(0.63-1.38)$ & 0.73 \\
\hline Collagen disease (vs. without) & $167(3.7)$ & 70.1 & $0.54(0.36-0.82)$ & 0.004 \\
\hline Rheumatoid arthritis (vs. without) & $112(2.5)$ & 73.2 & $0.61(0.36-1.03)$ & 0.06 \\
\hline Thyroid disease (vs. without) & $206(4.6)$ & 83.0 & $1.26(0.82-1.96)$ & 0.29 \\
\hline Liver disease (vs. without) & $227(5.1)$ & 76.2 & $0.65(0.45-0.94)$ & 0.02 \\
\hline COPD (vs. without) & $129(4.3)$ & 79.2 & $0.70(0.45-1.10)$ & 0.13 \\
\hline Gastroduodenal ulcer (vs. without) & $108(2.4)$ & 87.0 & $1.30(0.68-2.51)$ & 0.43 \\
\hline Depression (vs. without) & $103(2.3)$ & 81.6 & $1.38(0.74-2.60)$ & 0.31 \\
\hline Dementia (vs. without) & $52(1.16)$ & 98.1 & $3.94(0.50-31.0)$ & 0.19 \\
\hline Hemiplegia (vs. without) & $46(1.0)$ & 97.8 & $3.18(0.42-24.1)$ & 0.26 \\
\hline
\end{tabular}

Abbreviations: eGFR, estimated glomerular filtration rate; COPD, chronic obstructive pulmonary disease; CI, confidence interval.

Denominator was 4,476 patients.

Hypertension was defined as a systolic blood pressure $\geq 140 \mathrm{mmHg}$ or a diastolic blood pressure $\geq 90 \mathrm{mmHg}$ and/or current treatment with antihypertensive agents.

Diabetes mellitus was identified in patients with a hemoglobin A1c value $\geq 6.5 \%$ (National Glycohemoglobin Standardization Program), whose underlying kidney disease was diabetic nephropathy, and/or who were receiving treatment with insulin or oral antihyperglycemic drugs.

Dyslipidemia was defined as a triglyceride concentration $\geq 150 \mathrm{mg} / \mathrm{dL}$, a low-density lipoprotein cholesterol concentration $\geq 140 \mathrm{mg} / \mathrm{dL}$, a highdensity lipoprotein cholesterol concentration $<40 \mathrm{mg} / \mathrm{dL}$, or use of lipid-lowering medication.

Cardiovascular disease was defined as a composite of ischemic heart disease, congestive heart failure, ischemic stroke, hemorrhagic stroke, peripheral artery disease, thoracic aortic aneurysm, and abdominal aortic aneurysm.

Adjusted using the final selected model (age, sex, underlying kidney disease, hypertension, diabetes mellitus, dyslipidemia, history of cardiovascular disease, body mass index, and urinary protein excretion). Variables relevant to the categories were excluded from each model.

a $\mathrm{eGFR}<50 \mathrm{~mL} / \mathrm{min} / 1.73 \mathrm{~m}^{2}$ was the cutoff value.

conditions, 4.37; 95\% CI: 1.75-10.9). This upward trend was most pronounced with cardiovascular comorbidities (OR for 1-2 conditions, 2.34; 95\% CI, 1.76-3.11; OR for 3-4 conditions, 3.29; 95\% CI, 2.26-4.78; and OR for $\geq 5$ conditions, 7.12 ; $95 \%$ CI, 2.05-24.7). There was no significant association between the cumulative number of non-cardiovascular comorbidities and reduced eGFR ( $P$ for trend, 0.93). As Fig. 2 shows, the number of medications increased linearly with the progression of the CKD stage, and a significant dose-response relationship between polypharmacy and reduced eGFR was observed in the multivariable-adjusted model (Table 6).

\section{Discussion}

The FKR study is an observational cohort study of Japanese patients with non-dialysis-dependent CKD under nephrologist care. This cohort includes approximately 4,500 outpatients with CKD stages 
Table 5. The association between multimorbidity and reduced eGFR $\left(<60 \mathrm{~mL} / \mathrm{min} / 1.73 \mathrm{~m}^{2}\right)$

\begin{tabular}{|c|c|c|c|c|}
\hline & $\begin{array}{l}\text { No. (\%) of included } \\
\text { patients }\end{array}$ & $\begin{array}{l}\text { Prevalence of patients } \\
\text { with an eGFR }<60 \\
\mathrm{~mL} / \mathrm{min} / 1.73 \mathrm{~m}^{2}(\%)\end{array}$ & $\begin{array}{l}\text { Adjusted odds ratio } \\
\qquad(95 \% \mathrm{CI})\end{array}$ & $P$ value \\
\hline \multicolumn{5}{|c|}{ All 23 registered comorbidities, No. of conditions } \\
\hline 0 & $304(6.8)$ & 31.9 & 1.00 (reference) & \multirow{4}{*}{$<0.001$} \\
\hline $1-2$ & $2,286(51.1)$ & 71.3 & $2.11(1.54-2.91)$ & \\
\hline $3-4$ & $1,473(32.9)$ & 87.0 & $2.38(1.66-3.41)$ & \\
\hline$\geq 5$ & $413(9.2)$ & 92.7 & $2.94(1.74-4.97)$ & \\
\hline \multicolumn{5}{|c|}{ Major comorbidities, No. of conditions } \\
\hline 0 & $357(8.0)$ & 31.5 & 1.0 (reference) & \multirow{4}{*}{$<0.001$} \\
\hline $1-2$ & $2,652(59.2)$ & 72.9 & $2.22(1.65-2.97)$ & \\
\hline $3-4$ & $1,289(28.8)$ & 90.3 & $3.04(2.12-4.37)$ & \\
\hline$\geq 5$ & $178(4.0)$ & 96.7 & $4.37(1.75-10.9)$ & \\
\hline \multicolumn{5}{|c|}{ Cardiovascular comorbidities, No. of conditions } \\
\hline 0 & $382(8.5)$ & 33.0 & 1.00 (reference) & \multirow{4}{*}{$<0.001$} \\
\hline $1-2$ & $2,844(63.6)$ & 74.0 & $2.34(1.76-3.11)$ & \\
\hline $3-4$ & $1,110(24.8)$ & 91.4 & $3.29(2.26-4.78)$ & \\
\hline$\geq 5$ & $140(3.1)$ & 97.9 & $7.12(2.05-24.7)$ & \\
\hline \multicolumn{5}{|c|}{ Non-cardiovascular comorbidities, No. of conditions } \\
\hline 0 & $2,945(65.8)$ & 71.9 & 1.00 (reference) & \multirow{4}{*}{0.93} \\
\hline 1 & $1,112(24.8)$ & 83.5 & $1.09(0.88-1.36)$ & \\
\hline 2 & $325(7.3)$ & 82.5 & $0.80(0.56-1.14)$ & \\
\hline$\geq 3$ & $94(2.1)$ & 83.0 & $0.78(0.42-1.46)$ & \\
\hline
\end{tabular}

Abbreviations: eGFR, estimated glomerular filtration rate; CI, confidence interval.

Major comorbid conditions included hypertension, diabetes, dyslipidemia, prior cardiovascular disease, cancer, and bone fracture.

Adjusted using the final selected model, which included age, sex, underlying kidney disease, hypertension, diabetes mellitus, dyslipidemia, history of cardiovascular disease, body mass index, and urinary protein excretion. Variables relevant to the categories were excluded from each model.

Table 6. The association between the number of medications and reduced eGFR $\left(<60 \mathrm{~mL} / \mathrm{min} / 1.73 \mathrm{~m}^{2}\right)$

\begin{tabular}{lccc}
\hline No. of medications & No. (\%) of included patients & $\begin{array}{c}\text { Prevalence of patients with an } \\
\text { eGFR }<60 \mathrm{~mL} / \mathrm{min} / 1.73 \mathrm{~m}^{2}(\%)\end{array}$ & Adjusted odds ratio $(95 \% \mathrm{CI})$ \\
\hline 0 & $248(5.5)$ & 38.7 & 1.0 (reference) \\
$1-2$ & $882(18.4)$ & 56.6 & $1.29(0.87-1.92)$ \\
$3-4$ & $1,138(25.4)$ & 71.9 & $1.96(1.31-2.92)$ \\
$5-6$ & $997(22.3)$ & 83.8 & $2.36(1.54-3.62)$ \\
$\geq 7$ & $1,271(28.4)$ & 92.7 & $3.39(2.13-5.40)$ \\
\hline
\end{tabular}

Abbreviations: eGFR, estimated glomerular filtration rate; CI, confidence interval.

Adjusted using the final selected model, which included age, sex, underlying kidney disease, hypertension, diabetes mellitus, dyslipidemia, history of cardiovascular disease, body mass index, and urinary protein excretion.

G1-G5. A multivariable analysis revealed that lifestyle-related diseases, such as hypertension, diabetes mellitus, dyslipidemia, and a history of CVD, were independent factors associated with reduced kidney function. Interestingly, an independent linear association between the cumulative number of comorbidities and eGFR reduction was observed. The findings from this study provide a comprehensive understanding of demographics and highlight the importance of managing multimorbidity among patients with CKD under nephrologist care in Japan.

Patients with advanced CKD stages were older, more likely to have diabetes mellitus and cancer, and more likely to have high blood pressure levels, coronary artery disease, heart failure, and PAD. These data demonstrate the high disease burden of chronic diseases among older adults with comorbidities associated with CKD. Comorbidities, including CVD, are a major source of morbidity and healthcare costs for hospitalized elderly patients ${ }^{21)}$. Furthermore, 
CKD influences diagnosis and treatment decisions for these other comorbidities ${ }^{22)}$. Thus, early detection of $\mathrm{CKD}$ and appropriate management of chronic diseases may be a useful strategy to prevent adverse outcomes caused by these comorbidities.

A multivariable analysis revealed an independent association between reduced kidney function and comorbidities, including lifestyle-related disease and CVD risk factors. The high prevalence of cardiovascular risk factors in patients with advanced CKD suggests that the underlying cause of CKD may be a form of diffuse atherosclerotic vascular disease, as well as cardiac and other vascular diseases. This hypothesis is consistent with prior pathophysiological and epidemiological studies ${ }^{23,24)}$. The strong association between CKD and CVD suggests not only that both conditions share risks but also that CKD itself may promote a complex cardiovascular risk condition $5,25,26)$. Longitudinal investigations are needed to more fully explore this hypothesis.

Tonelli et al. assessed medical complexity using nine markers (including the number of comorbidities, number of prescription medications, presence of psychiatric disorders, number of types of physicians involved in each patient's care, and number of physicians involved in each patient's care) and found that patients with CKD had the highest complexity, leading to an increased risk of death ${ }^{9)}$. The authors concluded that intensive risk management is recommended for patients with $\mathrm{CKD}$ at high risk for comorbid CVD. However, such intensive management has a negative aspect, which may lead to complexity of care because of the increased number of medications. An increase in the number of medications can lead to adverse effects ${ }^{11)}$ and reduced kidney function due to poor adherence ${ }^{12)}$. Indeed, in our study, we found a significant dose-response relationship between the cumulative number of complications (especially cardiovascular complications) and reduced eGFR. These findings suggest that complications in patients with impaired kidney function are mainly due to increased cardiovascular burden, and therefore, an increase in the number of medications may have an unfavorable impact on kidney function through adverse effects and reduced adherence. A longitudinal study of our cohort needs to be further investigated to determine what practice patterns regarding medication use are protective for kidney outcomes.

Ethnic differences exist in terms of susceptibility to CVD. Previous epidemiological studies have found that Western populations have a higher incidence of ischemic heart diseases, such as myocardial infarction and angina pectoris ${ }^{27-29)}$. The crude prevalence of prior ischemic heart disease in our cohort was 10.8\%, similar to the $13.4 \%$ observed in the CKD-JAC study in Japan but less than the $26.0 \%$ observed in the Chronic Renal Insufficiency Cohort study in the United States $\left.{ }^{30}, 31\right)$. Previous stroke was found in $11.1 \%$ of patients in our cohort, similar to the CKDJAC study $(11.5 \%)$, but the prevalence of stroke in individuals with an eGFR $30-60 \mathrm{~mL} / \mathrm{min} / 1.73 \mathrm{~m}^{2}$ in the German CKD cohort deviated significantly to $37.2 \%{ }^{32}$. The incidence of CVD, including stroke, may be less common in Japanese patients with CKD compared with Caucasians. A prospective survey of our cohort will also provide new insights into ethnic differences in CVD burden among CKD populations.

Multivariable-adjusted models showed an inverse association between diabetes and reduced kidney function (eGFR $<60 \mathrm{~mL} / \mathrm{min} / 1.73 \mathrm{~m}^{2}$ ). To address multicollinearity, we performed a sensitivity analysis excluding diabetic nephropathy from the model, but the OR of diabetes for reduced GFR was not significant (OR, 1.05, 95\% CI, 0.85-1.30). We speculate that a possible explanation for these findings observed in this cross-sectional study is that the influence of diabetes may have been diluted by the uniqueness of the participants in this cohort, which was restricted to patients with CKD. Future longitudinal studies of this cohort will reveal the definitive association between diabetes and kidney function.

Our study has several limitations. First, we did not perform $\mathrm{Cr}$ clearance measurements or urine protein measurements through $24 \mathrm{~h}$ urine collection. However, given that routine practice is based on spot urinalysis, evidence based on opportunistic testing may be useful in real-world practice. Second, there is the possibility of misclassification of underlying kidney disease. Nevertheless, the biopsy diagnosis rate in our cohort was relatively high (37\%); thus, a highly accurate estimate could be expected. Finally, this study is cross-sectional and cannot address causality. A longitudinal study of this cohort should elucidate the association between comorbidities and the prognosis of Japanese patients with CKD.

\section{Conclusions}

In conclusion, $\mathrm{CKD}$ is associated with a higher prevalence of comorbid conditions. Importantly, patients with CKD are at a higher risk of comorbid CVD and require intensive monitoring and risk management. Based on these findings, emphasis should be placed on preventing adverse outcomes in patients with CKD through comprehensive multimorbidity risk management. We believe that this 
study could provide a better knowledge of demographics and multimorbidity among patients with CKD under nephrologist care in Japan.

\section{Authors' Contributions}

S.T. and T.N. contributed to the study design, acquisition of data, statistical analysis, interpretation of data, and drafting of the manuscript. H.H contributed to the study design, statistical analysis, interpretation of data, and drafting of the manuscript. K.T. and T.K. contributed to the critical revision of the manuscript and study supervision. All authors provided critical reviews of the manuscript and approved the final version.

\section{Acknowledgements}

The authors thank the participants in the FKR study, the members of the FKR Study Group, and all personnel at participating institutions involved in the study.

We thank Satoru Fujimi (Fukuoka Renal Clinic), Hideki Hirakata (Fukuoka Renal Clinic), Tadashi Hirano (Hakujyuji Hospital), Tetsuhiko Yoshida (Hamanomachi Hospital), Takashi Deguchi (Hamanomachi Hospital), Hideki Yotsueda (Harasanshin Hospital), Kiichiro Fujisaki (Iizuka Hospital), Keita Takae (Japanese Red Cross Fukuoka Hospital), Koji Mitsuiki (Japanese Red Cross Fukuoka Hospital), Akinori Nagashima (Japanese Red Cross Karatsu Hospital), Ritsuko Katafuchi (Kano Hospital), Hidetoshi Kanai (Kokura Memorial Hospital), Kenji Harada (Kokura Memorial Hospital), Tohru Mizumasa (Kyushu Central Hospital), Takanari Kitazono (Kyushu University), Toshiaki Nakano (Kyushu University), Toshiharu Ninomiya (Kyushu University), Kumiko Torisu (Kyushu University), Akihiro Tsuchimoto (Kyushu University), Shunsuke Yamada (Kyushu University), Hiroto Hiyamuta (Kyushu University), Shigeru Tanaka (Kyushu University), Dai Matsuo (Munakata Medical Association Hospital), Yusuke Kuroki (National Fukuoka-Higashi Medical Center), Hiroshi Nagae (National Fukuoka-Higashi Medical Center), Masaru Nakayama (National Kyushu Medical Center), Kazuhiko Tsuruya (Nara Medical University), Masaharu Nagata (Shin-eikai Hospital), Taihei Yanagida (Steel Memorial Yawata Hospital), Shotaro Onaka (Tagawa Municipal Hospital). We thank Emily Woodhouse, PhD, from Edanz Group (https:// en-author-services.edanz.com/ac) for editing a draft of this manuscript.

\section{Conflict of Interest}

The authors declare that they have no relevant

\section{References}

1) Imai $E$, Horio $M$, Iseki $K$, Yamagata $K$, Watanabe $T$, Hara S, Ura N, Kiyohara Y, Hirakata H, Moriyama T, Ando Y, Nitta K, Inaguma D, Narita I, Iso H, Wakai K, Yasuda Y, Tsukamoto Y, Ito S, Makino H, Hishida A, and Matsuo S: Prevalence of chronic kidney disease (CKD) in the Japanese general population predicted by the MDRD equation modified by a Japanese coefficient. Clin Exp Nephrol, 2007; 11: 156-163

2) Liyanage $T$, Ninomiya $T$, Jha $V$, Neal $B$, Patrice $H M$, Okpechi I, Zhao MH, Lv J, Garg AX, Knight J, Rodgers A, Gallagher M, Kotwal S, Cass A, and Perkovic V: Worldwide access to treatment for end-stage kidney disease: a systematic review. Lancet, 2015; 385: 19751982

3) Schieppati A, and Remuzzi G: Chronic renal diseases as a public health problem: epidemiology, social, and economic implications. Kidney Int Suppl, 2005; S7-S10

4) Ninomiya T, Kiyohara $Y$, Kubo M, Tanizaki Y, Doi Y, Okubo K, Wakugawa Y, Hata J, Oishi Y, Shikata K, Yonemoto K, Hirakata $\mathrm{H}$, and Iida M: Chronic kidney disease and cardiovascular disease in a general Japanese population: the Hisayama Study. Kidney Int, 2005; 68: 228-236

5) Go AS, Chertow GM, Fan D, McCulloch CE, and Hsu CY: Chronic kidney disease and the risks of death, cardiovascular events, and hospitalization. N Engl J Med, 2004; 351: 1296-1305

6) Yamada $S$, Tsuruya $K$, Taniguchi $M$, Tokumoto $M$, Fujisaki K, Hirakata H, Fujimi S, and Kitazono T: Association between serum phosphate levels and stroke risk in patients undergoing hemodialysis: the Q-cohort study. Stroke, 2016; 47: 2189-2196

7) Chronic Kidney Disease Prognosis Consortium; Matsushita K, van der Velde M, Astor BC, Woodward M, Levey AS, de Jong PE, Coresh J, and Gansevoort RT: Association of estimated glomerular filtration rate and albuminuria with all-cause and cardiovascular mortality in general population cohorts: a collaborative meta-analysis. Lancet, 2010; 375: 2073-2081

8) Hallan SI, Matsushita K, Sang Y, Mahmoodi BK, Black C, Ishani A, Kleefstra N, Naimark D, Roderick P, Tonelli M, Wetzels JF, Astor BC, Gansevoort RT, Levin A, Wen CP, Coresh J, and Chronic Kidney Disease Prognosis Consortium: Age and association of kidney measures with mortality and end-stage renal disease. JAMA, 2012; 308: 2349-2360

9) Tonelli M, Wiebe N, Manns BJ, Klarenbach SW, James MT, Ravani P, Pannu N, Himmelfarb J, and Hemmelgarn BR: Comparison of the complexity of patients seen by different medical subspecialists in a universal health care system. JAMA Netw Open, 2018; 1: e184852

10) Blydt-Hansen TD, Pierce CB, Cai Y, Samsonov D, Massengill S, Moxey-Mims M, Warady BA, and Furth SL: Medication treatment complexity and adherence in 
children with CKD. Clin J Am Soc Nephrol, 2014; 9: $247-254$

11) Kojima T, Akishita M, Kameyama Y, Yamaguchi K, Yamamoto H, Eto M, and Ouchi Y: High risk of adverse drug reactions in elderly patients taking six or more drugs: analysis of inpatient database. Geriatr Gerontol Int, 2012; 12: 761-762

12) Roy L, White-Guay B, Dorais M, Dragomir A, Lessard $\mathrm{M}$, and Perreault S: Adherence to antihypertensive agents improves risk reduction of end-stage renal disease. Kidney Int, 2013; 84: 570-577

13) Tanaka $S$, Ninomiya T, Fujisaki K, Yoshida H, Nagata $M$, Masutani K, Tokumoto M, Mitsuiki K, Hirakata H, Fujimi S, Kiyohara Y, Kitazono T, Tsuruya K, and Fukuoka Kidney disease Registry (FKR) Study Collaboration Group: The Fukuoka Kidney disease Registry (FKR) Study: design and methods. Clin Exp Nephrol, 2017; 21: 465-473

14) Kidney Disease: Improving Global Outcomes (KDIGO) CKD Work Group: KDIGO 2012 Clinical Practice Guideline for the Evaluation and Management of Chronic Kidney Disease. Kidney Int Suppl, 2013; 3: 1-150

15) Levey AS, Eckardt KU, Tsukamoto Y, Levin A, Coresh J, Rossert J, De Zeeuw D, Hostetter TH, Lameire N, and Eknoyan G: Definition and classification of chronic kidney disease: a position statement from Kidney Disease: Improving Global Outcomes (KDIGO). Kidney Int, 2005; 67: 2089-2100

16) Levin A, and Stevens PE: Summary of KDIGO 2012 CKD Guideline: behind the scenes, need for guidance, and a framework for moving forward. Kidney Int, 2014; 85: 49-61

17) Matsuo S, Imai E, Horio M, Yasuda Y, Tomita K, Nitta K, Yamagata K, Tomino Y, Yokoyama H, and Hishida A: Revised equations for estimated GFR from serum creatinine in Japan. Am J Kidney Dis, 2009; 53: 982-992

18) Schwartz GJ, Haycock GB, Edelmann CM, Jr., and Spitzer A: A simple estimate of glomerular filtration rate in children derived from body length and plasma creatinine. Pediatrics, 1976; 58: 259-263

19) Schwartz GJ, Brion LP, and Spitzer A: The use of plasma creatinine concentration for estimating glomerular filtration rate in infants, children, and adolescents. Pediatr Clin North Am, 1987; 34: 571-590

20) Charlson ME, Pompei P, Ales KL, and MacKenzie CR: A new method of classifying prognostic comorbidity in longitudinal studies: development and validation. J Chronic Dis, 1987; 40: 373-383

21) Steinman MA, Landefeld CS, Rosenthal GE, Berthenthal D, Sen S, and Kaboli PJ: Polypharmacy and prescribing quality in older people. J Am Geriatr Soc, 2006; 54: 1516-1523

22) Dukkipati R, Adler S, and Mehrotra R: Cardiovascular implications of chronic kidney disease in older adults. Drugs Aging, 2008; 25: 241-253
23) Kasiske BL: Relationship between vascular disease and age-associated changes in the human kidney. Kidney Int, 1987; 31: 1153-1159

24) Anderson S, and Brenner BM: Effects of aging on the renal glomerulus. Am J Med, 1986; 80: 435-442

25) Anavekar NS, McMurray JJ, Velazquez EJ, Solomon SD, Kober L, Rouleau JL, White HD, Nordlander R, Maggioni A, Dickstein K, Zelenkofske S, Leimberger JD, Califf RM, and Pfeffer MA: Relation between renal dysfunction and cardiovascular outcomes after myocardial infarction. N Engl J Med, 2004; 351: 1285-1295

26) Astor BC, Hallan SI, Miller ER, 3rd, Yeung E, and Coresh J: Glomerular filtration rate, albuminuria, and risk of cardiovascular and all-cause mortality in the US population. Am J Epidemiol, 2008; 167: 1226-1234

27) McCullough PA, Li S, Jurkovitz CT, Stevens LA, Wang C, Collins AJ, Chen SC, Norris KC, McFarlane SI, Johnson B, Shlipak MG, Obialo CI, Brown WW, Vassalotti JA, Whaley-Connell AT, and Kidney Early Evaluation Program Investigators: CKD and cardiovascular disease in screened high-risk volunteer and general populations: the Kidney Early Evaluation Program (KEEP) and National Health and Nutrition Examination Survey (NHANES) 1999-2004. Am J Kidney Dis, 2008; 51: S38-45

28) Thrift AG, Cadilhac DA, Thayabaranathan T, Howard G, Howard VJ, Rothwell PM, and Donnan GA: Global stroke statistics. Int J Stroke, 2014; 9: 6-18

29) Nowbar AN, Howard JP, Finegold JA, Asaria P, and Francis DP: 2014 global geographic analysis of mortality from ischaemic heart disease by country, age and income: statistics from World Health Organisation and United Nations. Int J Cardiol, 2014; 174: 293-298

30) Lash JP, Go AS, Appel LJ, He J, Ojo A, Rahman M, Townsend RR, Xie D, Cifelli D, Cohan J, Fink JC, Fischer MJ, Gadegbeku C, Hamm LL, Kusek JW, Landis JR, Narva A, Robinson N, Teal V, Feldman HI, and Chronic Renal Insufficiency Cohort (CRIC) Study Group: Chronic Renal Insufficiency Cohort (CRIC) Study: baseline characteristics and associations with kidney function. Clin J Am Soc Nephrol, 2009; 4: 13021311

31) Imai $E$, Matsuo $S$, Makino $H$, Watanabe $T$, Akizawa $T$, Nitta K, Iimuro S, Ohashi Y, and Hishida A: Chronic Kidney Disease Japan Cohort study: baseline characteristics and factors associated with causative diseases and renal function. Clin Exp Nephrol, 2010; 14: 558-570

32) Titze S, Schmid M, Kottgen A, Busch M, Floege J, Wanner C, Kronenberg F, Eckardt KU, and GCKD study investigators: Disease burden and risk profile in referred patients with moderate chronic kidney disease: composition of the German Chronic Kidney Disease (GCKD) cohort. Nephrol Dial Transplant, 2015; 30: 441-451 
Supplementary Table 1. Baseline treatment status of the participants according to CKD stages

\begin{tabular}{|c|c|c|c|c|c|c|c|}
\hline & All patients & Stage G1-2 & Stage G3a & Stage G3b & Stage G4 & Stage G5 & $P$ value \\
\hline \multicolumn{8}{|l|}{ Antihypertensive agents } \\
\hline ACE inhibitors, \% & 9.1 & 6.7 & 10.1 & 11.3 & 10.2 & 6.6 & 0.30 \\
\hline $\mathrm{ARBs}, \%$ & 65.4 & 50.7 & 61.2 & 68.2 & 75.8 & 79.6 & $<0.001$ \\
\hline Combined ACE inhibitors and ARBs, \% & 4.4 & 3.0 & 5.1 & 5.2 & 4.7 & 4.3 & 0.13 \\
\hline Calcium channel blockers, $\%$ & 48.7 & 24.6 & 38.4 & 52.3 & 64.9 & 75.1 & $<0.001$ \\
\hline Beta blockers, $\%$ & 15.2 & 4.4 & 10.0 & 15.8 & 23.2 & 28.0 & $<0.001$ \\
\hline Alfa blockers, $\%$ & 6.1 & 1.2 & 2.5 & 5.5 & 9.5 & 16.0 & $<0.001$ \\
\hline Diuretics, \% & 21.1 & 4.7 & 11.2 & 22.9 & 33.7 & 41.1 & $<0.001$ \\
\hline \multicolumn{8}{|c|}{ Lipid-, glucose-, and uric acid-lowering therapy and antiplatelet/anticoagulant therapy } \\
\hline Statins, $\%$ & 40.2 & 31.5 & 40.7 & 45.5 & 42.7 & 43.0 & $<0.001$ \\
\hline Insulin, $\%$ & 6.4 & 1.8 & 2.9 & 5.6 & 9.4 & 16.6 & $<0.001$ \\
\hline Sulfonylureas, \% & 3.0 & 0.7 & 2.8 & 4.6 & 4.4 & 2.3 & $<0.001$ \\
\hline Biguanides, $\%$ & 1.6 & 1.9 & 2.2 & 2.4 & 0.9 & 0.0 & 0.002 \\
\hline$\alpha$-glucosidase inhibitors, $\%$ & 4.9 & 1.6 & 3.1 & 6.3 & 7.0 & 7.6 & $<0.001$ \\
\hline Dipeptidyl peptidase- 4 inhibitors, $\%$ & 12.7 & 5.3 & 9.2 & 12.9 & 18.7 & 20.6 & $<0.001$ \\
\hline Uric acid-lowering agents, $\%$ & 41.1 & 9.8 & 27.2 & 45.1 & 67.1 & 67.2 & $<0.001$ \\
\hline Antiplatelets, \% & 18.3 & 4.5 & 10.7 & 20.5 & 29.6 & 31.1 & $<0.001$ \\
\hline Warfarin, \% & 6.0 & 2.3 & 3.7 & 7.4 & 9.8 & 7.0 & $<0.001$ \\
\hline \multicolumn{8}{|l|}{ Treatment for anemia } \\
\hline ESAs, \% & 14.0 & 0.2 & 1.1 & 6.3 & 23.0 & 57.6 & $<0.001$ \\
\hline Iron, \% & 7.3 & 3.5 & 3.6 & 5.1 & 10.6 & 18.3 & $<0.001$ \\
\hline \multicolumn{8}{|c|}{ Treatment for MBD, acidosis, uremic toxin, and hyperkalemia } \\
\hline Phosphate binders, \% & 1.4 & 0.1 & 0.4 & 0.5 & 0.9 & 8.1 & $<0.001$ \\
\hline Vitamin D3, \% & 10.8 & 11.0 & 8.3 & 9.0 & 11.0 & 17.4 & 0.002 \\
\hline Sodium bicarbonate, $\%$ & 4.6 & 0.0 & 0.5 & 1.4 & 7.5 & 20.2 & $<0.001$ \\
\hline Carbonic absorbent, \% & 4.9 & 0.0 & 0.5 & 2.4 & 7.6 & 21.1 & $<0.001$ \\
\hline Ion exchange resins, $\%$ & 11.3 & 0.3 & 2.1 & 5.4 & 20.6 & 39.6 & $<0.001$ \\
\hline
\end{tabular}

Abbreviations: CKD, chronic kidney disease; ARBs, Angiotensin II receptor blockers; ACE, angiotensin-converting enzyme; ESAs, erythropoiesisstimulating agents; MBD, mineral bone disorders. Values are given as percentage. 
Supplementary Table 2. Prevalence of all registered 23 comorbidities of the participants according to CKD stages

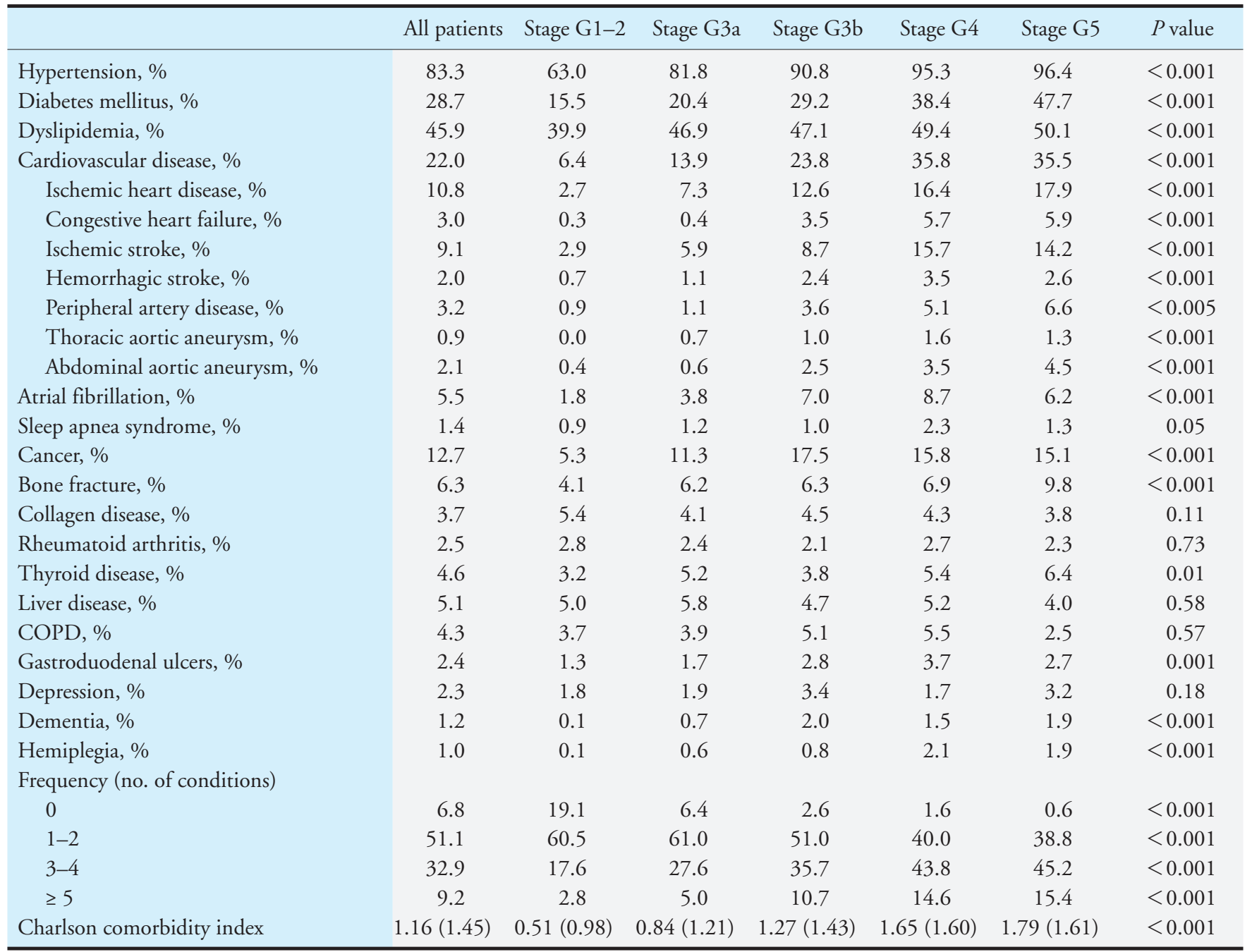

Abbreviations: CKD, chronic kidney disease; COPD, chronic obstructive pulmonary disease. Values are presented as mean (standard deviation) or percentage. Hypertension was defined as a systolic blood pressure $\geq 140 \mathrm{mmHg}$ or a diastolic blood pressure $\geq 90 \mathrm{mmHg}$ and/or current use of antihypertensive agents. Diabetes mellitus was identified in patients with a hemoglobin A1c value $\geq 6.5 \%$ (National Glycohemoglobin Standardization Program), whose underlying kidney disease was diabetic nephropathy, and/or who received treatment with insulin or oral antihyperglycemic drugs.

Dyslipidemia was defined as a triglyceride concentration $\geq 150 \mathrm{mg} / \mathrm{dL}$, a low-density lipoprotein cholesterol concentration $\geq 140 \mathrm{mg} / \mathrm{dL}$, a highdensity lipoprotein cholesterol concentration $<40 \mathrm{mg} / \mathrm{dL}$, or use of lipid-lowering medication.

Cardiovascular disease was defined as a composite of ischemic heart disease, congestive heart failure, ischemic stroke, hemorrhagic stroke, peripheral artery disease, thoracic aortic aneurysm, and abdominal aortic aneurism. 
Supplementary Table 3. The association between multimorbidity and reduced eGFR $\leq 45$ and $\leq 30 \mathrm{~mL} / \mathrm{min} / 1.73 \mathrm{~m}^{2}$

\begin{tabular}{|c|c|c|c|c|c|c|c|}
\hline \multirow[b]{2}{*}{ No. of comorbid conditions } & \multirow[b]{2}{*}{$\begin{array}{l}\text { No. (\%) of included } \\
\text { subjects }\end{array}$} & \multicolumn{3}{|c|}{$\mathrm{eGFR} \leq 45 \mathrm{~mL} / \mathrm{min} / 1.73 \mathrm{~m}^{2}$} & \multicolumn{3}{|c|}{$\mathrm{eGFR} \leq 30 \mathrm{~mL} / \mathrm{min} / 1.73 \mathrm{~m}^{2}$} \\
\hline & & $\begin{array}{l}\text { Prevalence of subjects } \\
\text { with eGFR } \leq 45 \mathrm{~mL} / \\
\min / 1.73 \mathrm{~m}^{2}(\%)\end{array}$ & $\begin{array}{l}\text { Adjusted odds ratio } \\
\quad(95 \% \mathrm{CI})\end{array}$ & $P$ value & $\begin{array}{c}\text { Prevalence of subjects } \\
\text { with eGFR } \leq 30 \mathrm{~mL} / \\
\min / 1.73 \mathrm{~m}^{2}(\%)\end{array}$ & $\begin{array}{l}\text { Adjusted odds ratio } \\
\qquad(95 \% \mathrm{CI})\end{array}$ & $P$ value \\
\hline \multicolumn{8}{|l|}{ All registered 23 comorbidities } \\
\hline 0 & $304(6.8)$ & 14.5 & 1.0 (reference) & \multirow{4}{*}{$<0.001$} & 6.6 & 1.0 (reference) & \multirow{4}{*}{$<0.001$} \\
\hline $1-2$ & $2,286(51.1)$ & 51.1 & $2.63(1.80-3.84)$ & & 28.2 & $3.71(2.17-6.86)$ & \\
\hline $3-4$ & $1,473(32.9)$ & 71.4 & $3.46(2.33-5.14)$ & & 48.8 & $5.05(2.92-9.40)$ & \\
\hline$\geq 5$ & $413(9.2)$ & 82.6 & $4.94(3.08-7.93)$ & & 58.4 & $5.85(3.27-11.2)$ & \\
\hline \multicolumn{8}{|l|}{ Major comorbidities } \\
\hline 0 & $357(8.0)$ & 15.4 & 1.0 (reference) & \multirow{4}{*}{$<0.001$} & 7.0 & 1.0 (reference) & \multirow{4}{*}{$<0.001$} \\
\hline $1-2$ & $2,652(59.2)$ & 51.4 & $2.91(2.07-4.10)$ & & 30.1 & $4.21(2.51-7.08)$ & \\
\hline $3-4$ & $1,289(28.8)$ & 75.7 & $4.25(2.93-6.15)$ & & 52.5 & $5.89(3.45-10.1)$ & \\
\hline$\geq 5$ & $178(4.0)$ & 92.7 & $11.7(5.76-23.73)$ & & 69.1 & $8.77(4.66-16.5)$ & \\
\hline \multicolumn{8}{|l|}{ Cardiovascular comorbidities } \\
\hline 0 & $382(8.5)$ & 17.0 & 1.0 (reference) & \multirow{4}{*}{$<0.001$} & 7.6 & 1.0 (reference) & \multirow{4}{*}{$<0.001$} \\
\hline $1-2$ & $2,844(63.6)$ & 52.6 & $2.79(2.03-3.84)$ & & 30.8 & $3.86(2.41-6.18)$ & \\
\hline $3-4$ & $1,110(24.8)$ & 78.0 & $4.32(3.01-6.18)$ & & 55.3 & $5.93(3.62-9.69)$ & \\
\hline$\geq 5$ & $140(3.1)$ & 94.3 & $15.5(6.52-37.0)$ & & 74.3 & $11.3(6.07-21.7)$ & \\
\hline \multicolumn{8}{|l|}{ Non-cardiovascular comorbidities } \\
\hline 0 & $2,945(65.8)$ & 52.6 & 1.0 (reference) & \multirow{4}{*}{0.88} & 33.2 & 1.0 (reference) & \multirow{4}{*}{0.42} \\
\hline 1 & $1,112(24.8)$ & 66.7 & $1.16(0.97-1.39)$ & & 42.7 & $1.01(0.86-1.20)$ & \\
\hline 2 & $325(7.3)$ & 62.5 & $0.79(0.59-1.04)$ & & 39.4 & $0.82(0.62-1.08)$ & \\
\hline$\geq 3$ & $94(2.1)$ & 69.2 & $1.09(0.65-1.84)$ & & 45.7 & $0.99(0.61-1.60)$ & \\
\hline
\end{tabular}

Abbreviations: eGFR, estimated glomerular filtration rate; $\mathrm{CI}$, confidence interval.

Major comorbid conditions included hypertension, diabetes, dyslipidemia, prior cardiovascular disease, cancer, and bone fracture.

Adjusted using the final selected model, which included age, sex, underlying kidney disease, hypertension, diabetes mellitus, dyslipidemia, history of cardiovascular disease, body mass index, and urinary protein excretion.

The variable relevant to the categories was excluded from each model. 


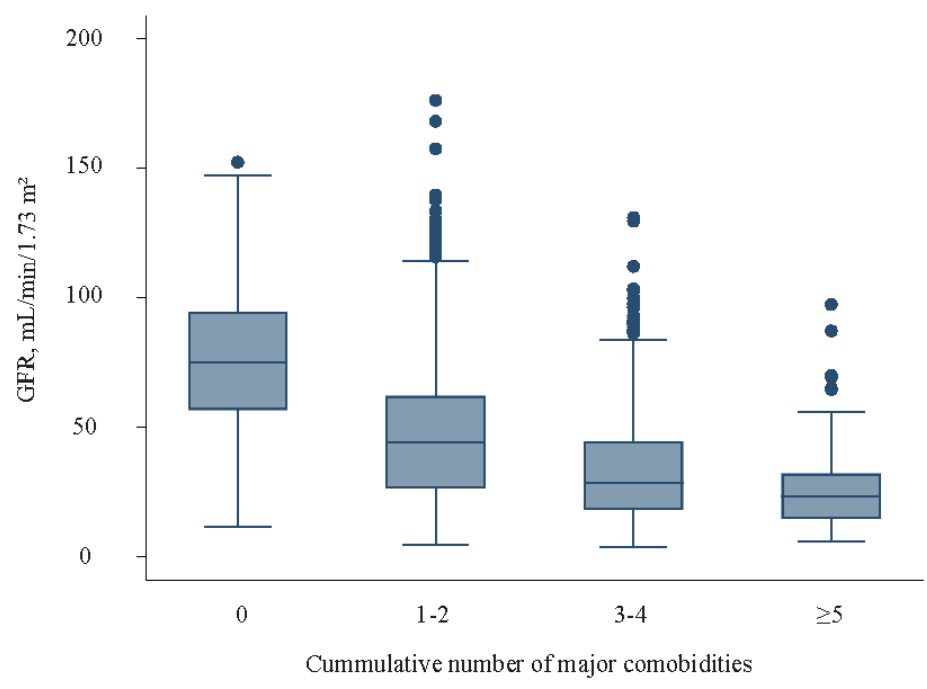

Supplementary Fig. 1. Box-and-whisker plots showing the association between the cumulative number of major comorbidities and eGFR Abbreviation: GFR, glomerular filtration rate.

Supplementary Table 4. The association between major comorbidities and eGFR in multivariable-adjusted linear regression model

\begin{tabular}{|c|c|c|c|c|c|}
\hline \multirow{2}{*}{$\begin{array}{l}\text { Major comorbidities, } \\
\text { no. of conditions }\end{array}$} & 0 & $1-2$ & $3-4$ & $\geq 5$ & \multirow{2}{*}{$P$ value } \\
\hline & Mean $(95 \% \mathrm{CI})$ & Mean $(95 \% \mathrm{CI})$ & Mean $(95 \% \mathrm{CI})$ & Mean $(95 \% \mathrm{CI})$ & \\
\hline \multicolumn{6}{|l|}{ Unadjusted } \\
\hline $\begin{array}{l}\text { eGFR, } \mathrm{mL} / \mathrm{min} / 1.73 \mathrm{~m}^{2} \\
\text { Adjusted }\end{array}$ & $74.9(72.1-77.7)$ & $46.5(45.5-47.5)$ & $33.2(32.1-34.3)$ & $25.8(23.7-27.9)$ & $<0.001$ \\
\hline $\mathrm{eGFR}, \mathrm{mL} / \mathrm{min} / 1.73 \mathrm{~m}^{2}$ & $57.8(55.5-60.1)$ & $44.0(43.2-44.7)$ & $40.9(39.8-42.0)$ & $39.4(36.4-42.3)$ & $<0.001$ \\
\hline
\end{tabular}

Abbreviations: eGFR, estimated glomerular filtration rate; CI, confidence interval.

Major comorbid conditions included hypertension, diabetes, dyslipidemia, prior cardiovascular disease, cancer, and bone fracture. Adjusted for age, sex, underlying kidney disease, body mass index, and urinary protein excretion. 